\title{
Appreciation of Incisive Foramen in Intraoral Periapical Radiographs - A Comparative Radiographic Study
}

\author{
K. M. Veena, ${ }^{1}$ Rameesa Razak, ${ }^{1}$ Laxmikanth Chatra, ${ }^{1}$ Prashanth Shenoy, ${ }^{1}$ Rachana V. Prabhu ${ }^{1}$ \\ 'Department of Oral Medicine and Radiology, Yenepoya University Yenepoya Dental College, Deralakatte, Mangalore, India
}

Disclose and conflicts of interest: none to be declared by all authors

\begin{abstract}
Introduction: the incisive foramen is said to be a normal anatomical landmark in intraoral periapical (IOPA) radiographs. The present study was performed to appreciate the visibility of the incisive foramen in routine periapical radiographs and to compare its visibility in bisecting angle and paralleling angle techniques.

Materials and Methods: the current study evaluated 120 IOPA radiographs of maxillary central. incisors. These was scored for visibility of incisive foramen by a radiographic expert.

Results: the study comprised of 60 bisecting and 60 paralleling radiographs of maxillary incisor teeth. Out of 60 bisecting radiographs, the incisive foramen was visible in only 24 radiographs, and there was no visibility of incisive foramen in 36 bisecting radiographs. However, in the 60 paralleling radiographs, the incisive foramen was visible in 46 radiographs and not visible in only 14 radiographs. The total percentage of visibility of the incisive foramen in the bisecting and paralleling radiographs was $40 \%$ and $76.7 \%$, respectively.

Conclusion: the incisive foramen, although considered to be a normal anatomical landmark seen in IOPA radiographs, is often not visible. Its exact location, morphology, and dimension are important for any surgical procedure in that site, especially for the placement of implants. The nasopalatine canal cyst, which is usually asymptomatic, is often a missed finding on radiographic examinations due to its similarity in appearance to the foramen. The understanding of visibility of the incisive foramen in routine intraoral radiographs is very important to the clinician. Our study shows that the paralleling technique offers better visibility when compared with the bisecting angle technique. Hence, the paralleling technique should be favored to visualize the foramen.
\end{abstract}

Keywords: Bisecting; Incisive foramen; Intraoral periapical radiographs; Paralleling; Visibility

\section{Introduction}

Diagnostic intraoral periapical (IOPA) radiography has evolved as an inseparable branch of dentistry. Proper IOPA technique not only helps to confirm the diagnosis but also aids treatment planning and management. It provides the baseline for assessing the outcome of each pulpal, periapical and periodontal pathologies. ${ }^{1}$ Periodic and comprehensive oral evaluations at dental offices frequently employ the use of radiographs to help visualize teeth and bones in ways that are not possible via the naked eye. Since that time, many improvements to the delivery of X-ray radiation have also been accomplished, and today, exposure has been minimized dramatically. In fact, digital X-rays, which deliver even less radiation than traditional film-based X-rays, have quickly become commonplace in dental offices throughout the world.

A successful interpretation of radiographs begins with an understanding of the normal anatomical landmarks. ${ }^{2}$ The incisive foramen is said to be a normal anatomical landmark seen in IOPA radiographs. It is usually an oval-shaped opening that faces the posteroinferior side of the palate. The diameter varies from 2 to $10 \mathrm{~mm}$, with a position that varies from just above the crest of the alveolar ridge to the level of the apices of the central incisors. ${ }^{2}$

The incisive foramen is actually in the anterior part of the palate, but superimposition makes it appear to be located between the roots of the central incisors. The radiographic position of the nasopalatine foramen may vary from just above the top of the alveolar crest to the level of the apices of the teeth because there are anatomic size variations and the X-rays have different vertical angulations. In some cases, the shadow of the foramen may be superimposed on the apex of a central incisor, which may be misinterpreted as periapical disease. It may appear smoothly symmetric, with numerous forms, or very irregular, with a well demarcated or ill-defined border. ${ }^{2}$

The visibility depends on its size, shape, location, and superimposition. This may be the result of the projection geometry and the variability in anatomic morphology. On two-dimensional radiographic exams, the image of the inci-sive foramen is usually projected between the roots of the central incisors.

The exact location, its morphology as well as dimension is important for any surgical procedure in that site, especially placement of implants. The 
nasopalatine canal cyst, usually asymptomatic are often missed in radiographic finding due to its appearance similar to foramen.

The exact location, its morphology as well as dimension is important for any surgical procedure in that site, especially placement of implants. The nasopalatine canal cyst, usually asymptomatic are often missed in radiographic finding due to its appearance similar to foramen.

Thus, this study is aimed to appreciate the visibility of the incisive foramen in routine periapical radiographs and to compare its visibility in bisecting angle and paralleling angle techniques.

\section{Materials and Methods}

The study was conducted in the Department of Oral Medicine \& Radiology, Yenepoya Dental College \& Hospital, Yenepoya University, Mangalore. After obtaining ethical clearance from the institutions ethical committee, 120 IOPA radiographs of maxillary central incisors, taken using digital radiography, were collected from the department database. Any faulty radiographs, radiographs with pathology of central incisors and of individuals with developmental anomalies, such as cleft palate, were excluded.

The incisive foramina were analyzed by a radiographic expert in the department.

\section{Results}

Table 1 - Shows the comparison between the visibility and non-visibility of the incisive foramen in bisecting and paralleling IOPA radiographs of maxillary incisors. The study comprised of 60 bisecting and 60 paralleling radiographs of maxillary incisor teeth. Out of 60 bisecting radiographs, the incisive foramen was visible in only 24 radiographs, and there was no visibility of the incisive foramen in 36 bisecting radiographs. However, in the 60 paralleling radiographs, the incisive foramen was visible in 46 radiographs and not visible in only 14 radiographs. The total percentage of visibility of the incisive foramen in the bisecting and paralleling radiographs was $40 \%$ and $76.7 \%$, respectively.

Table 2 - The Chi-squared test shows that there is a statistically significant difference between the two techniques with the $p$-value of 0.000 .

Table 1. Comparison between the visibility of the incisive foramen in bisecting and paralleling techniques

\begin{tabular}{l|c|c|c}
\hline \multirow{2}{*}{$\begin{array}{c}\text { Bisecting } \\
\text { Technique }\end{array}$} & \multicolumn{2}{|c|}{ Paralleling Technique } & \multirow{2}{*}{ Total } \\
\cline { 2 - 3 } & Not Visible & Visible & \\
\hline Not visible & 13 & 23 & 36 \\
\hline Visible & 1 & 23 & 24 \\
\hline Total & 14 & 46 & 60 \\
\hline
\end{tabular}

Table 2. Chi-squared test

\begin{tabular}{l|c|c}
\hline & Value & Exact Sig. (2-sided) \\
\hline McNemar test & & $0.000^{\mathrm{a}}$ \\
\hline Number of valid cases & 60 & \\
\hline
\end{tabular}

${ }^{a}$ Binomial distribution used.

\section{Discussion}

Imaging is an integral part of the diagnostic process in clinical dentistry. Radiographs are often obtained as part of a complete examination. Appropriate radiographic interpretation, along with clinical information and other tests, is used to formulate a differential diagnosis. Knowledge of normal radiographic anatomy is paramount to distinguish normal anatomy from variations of normal anatomy and pathologies.

The incisive foramen (also known as nasopalatine foramen or anterior palatine foramen) is the oral opening of the nasopalatine canal. It is located in the maxilla in the incisive fossa, midline in the palate posterior to the central incisors, at the junction of the medial palatine and incisive sutures. Radiographically, the incisive foramina appear as ovoid radiolucencies between the roots of the central incisors. ${ }^{3}$

The incisive foramen is said to be a normal anatomical landmark seen in IOPA radiographs. The visibility depends on its size, shape, location, and superimposition. This may be the result of the projection geometry and the variability in anatomic morphology.

The present study was done to evaluate the visibility of the incisive foramen in IOPA radiographs. A total of 120 radiographs of the maxillary anterior region, 60 bisecting radiographs and 60 paralleling radiographs, was used for the study.

The incisive foramenwas visible around $76.7 \%$ of paralleling radiographs, while it was visible in only $40 \%$ of bisecting radiographs.

The incisive foramen is actually located in the anterior part of the palate, but superimposition makes it appear to be located between the roots of the central incisors. The incisive canal connects the roof of the oral cavity with the floor of nasal cavity and has the incisive and nasal foramina respectively at its two opposite ends. Its close proximity with the anterior incisors affects one's ability to place immediate implants in ideal position. ${ }^{4}$

The nasopalatine canal cyst, which is normally asymptomatic, constitutes casual radiological findings. Radiographic interpretation should be thoroughly performed for maxillary anterior region and any occlusal radiograph in routine radiography to screen this entity and must be distinguished from the incisive foramen. A foramen larger than $6 \mathrm{~mm}$ may simulate the appearance of a cyst. ${ }^{5}$

Variations in the vertical angulations of X-rays and in the anatomic size of the incisive foramen results in 
the radiographic position of the nasopalatine foramen from just above the top of the alveolar crest at the level of the apices of the teeth. In some cases, the shadow of the foramen may be superimposed on the apex of a central incisor, a situation that requires attention to differentiate it from periapical diseases. This is done by taking another radiograph at a different horizontal angulation or by testing pulp vitality.

Variations in the vertical angulations of X-rays and in the anatomic size of the incisive foramen results in the radiographic position of the nasopalatine foramen from just above the top of the alveolar crest at the level of the apices of the teeth. In some cases, the shadow of the foramen may be superimposed on the apex of a central incisor, a situation that requires attention to differentiate it from periapical diseases. This is done by taking another radiograph at a different horizontal angulation or by testing pulp vitality.

The great variability in radiographic imaging is primarily the result of the different angles at which the X-ray beam is directed at the maxillary central incisors.

Although it is said to be a normal anatomical landmark that can be seen in most of the radiographs, the present study reveals that the incisive foramen is not visible in all routine radiographs that were taken in the south Indian population.

A similar study was conducted by Basappa S. et al in 200 orthopantomographs. This study concluded that the incisive foramen showed good visibility in only $25 \%$ of the orthopantomographs. The literature does not provide similar studies in periapical radiographs; hence, it cannot be generalized.

\section{Conclusions}

The incisive foramen, although considered to be a normal anatomical landmark seen in IOPA radiographs, is often not visible. The morphology and the dimensions of the incisive foramen show variations. Its exact location, morphology, and dimension are important for any surgical procedure in that site, especially for the placement of implants. The nasopalatine canal cyst, which is usually asymptomatic, is often a missed finding in radiographic examinations due to its similarity in appearance to the foramen. The understanding of the visibility of the incisive foramen in routine intraoral radiographs is very important to the clinician. Our study shows that the paralleling technique offers better visibility when compared with the bisecting angle technique. Hence, the paralleling technique should be favored to visualize the foramen.

\section{References}

1. Gupta A, Devi P, Srivastava R, Jyoti B. Intra oral periapical radiography - basics yet intrigue: A review. Bangla J Dent Res Educ 2014;4(02):83-87.

2. Georgescu CE, Tãnase G, Mihai A. Normal Radiographic Anatomy. Maxillary Central Area 2007;3:9.

3. Mudgal P. Incisive foramen | Radiology Reference Article | Radiopaedia.org. Radiopaedia. https://radiopaedia.org/articles/ inci- sive-foramen. Accessed August 19, 2019.

4. Al-Amery SM, Nambiar P, Jamaludin M, John J, Ngeow WC. Cone beam computed tomography assessment of the maxillary incisive canal and foramen: considerations of anatomical variations when placing immediate implants. PLoS One 2015;10(02): e0117251.

5. Srivastava S, Misra N, Agarwal R, Pandey P. Nasopalatine canal cyst: often missed. BMJ Case Rep 2013;2013:1-3.

6. Panjnoush M, Norouzi H, Kheirandish Y, Shamshiri AR, Mofidi
N. Evaluation of Morphology and Anatomical Measurement of Nasopalatine Canal Using Cone Beam Computed Tomography. J Dent (Tehran) 2016;13(04):287-294.

7. Rad AB, Forouzanfar A, Aghasizadeh E, et al. Radiographic evaluation of the incisive foramen position by Cone-Beam Computed Tomography (CBCT) in edentulous anterior maxilla regions and its relationship to dental implant placement of incisor teeth. Bali Med J 2017;6(02):236.

8. Khanum N. Visibility of Maxillary and Mandibular Anatomical Landmarks in Digital Panoramic Radiographs: A Retrospective Study. J Dent Sci 2017;5(03):6.

9. Alcaraz M, Parra C, Martínez Beneyto Y, Velasco E, Canteras M. Is it true that the radiation dose to which patients are exposed has decreased with modern radiographic films? Dentomaxillofac Radiol 2009;38(02):92-97.
Received: July 11, 2019

Accepted: September 16, 2019
Corresponding author

Veena K. M.

Email: veenaomr@gmail.com 